\title{
The prognostic significance of CD11b+CX3CR1+ monocytes in patients with newly diagnosed diffuse large B-cell lymphoma
}

\author{
Ho-Young Yhim ${ }^{1,2, *}$, Jeong-A Kim ${ }^{3,4, *}$, Sun-Hye Ko ${ }^{3}$, Youngrok Park ${ }^{5}$, Eunjung Yim ${ }^{3}$, \\ Hee Sun Kim ${ }^{6}$ and Jae-Yong Kwak ${ }^{1}$ \\ ${ }^{1}$ Department of Internal Medicine, Chonbuk National University Medical School, Jeonju, Republic of Korea \\ ${ }^{2}$ Research Institute of Clinical Medicine, Chonbuk National University-Biomedical Research Institute of Chonbuk National \\ University Hospital, Jeonju, Republic of Korea \\ ${ }^{3}$ Division of Hematology, Department of Internal Medicine, College of Medicine, The Catholic University of Korea, Seoul, \\ Republic of Korea \\ ${ }^{4}$ Leukemia Research Institute, The Catholic University of Korea, Seoul, Republic of Korea \\ ${ }^{5}$ Tumor Biology Training Program, Lombardi Comprehensive Cancer Center, Georgetown University School of Medicine, \\ Washington, DC, USA \\ ${ }^{6}$ College of Nursing, Chonbuk National University, Jeonju, Republic of Korea \\ *These authors have contributed equally to this work \\ Correspondence to: Jae-Yong Kwak, email: jykwak@jbnu.ac.kr \\ Keywords: angiogenesis, CD 1 1b, CX3CR 1, diffuse large B-cell lymphoma, immunosuppression \\ Received: May 14, $2017 \quad$ Accepted: August 17, $2017 \quad$ Published: September 23, 2017 \\ Copyright: Yhim et al. This is an open-access article distributed under the terms of the Creative Commons Attribution License 3.0 \\ (CC BY 3.0), which permits unrestricted use, distribution, and reproduction in any medium, provided the original author and source \\ are credited.
}

\section{ABSTRACT}

Despite their critical roles in angiogenesis and host immunosuppression within the tumor microenvironment, the prognostic significance of myeloid-lineage cells expressing CD11b and CX3CR1 in diffuse large B-cell lymphoma (DLBCL) has not been well studied. We prospectively enrolled newly-diagnosed DLBCL patients at two Korean institutions between May 2011 and Aug 2015. CD11b+CX3CR1 ${ }^{+}$cells were analyzed by flow cytometry using peripheral blood (PB) and bone marrow (BM) aspirate samples before treatments. Eighty-nine patients ( 52 males) were enrolled. The median age was 65 years (range, 19-88 years). Thirty-seven patients $(42 \%)$ were classified as high-intermediate or high risk according to the National Comprehensive Cancer Network International Prognostic Index (NCCN-IPI). Patients were categorized into either high or low PB-/BM-CD11 b+CX3CR1+ monocyte group according to the cutoffs identified by the receiver-operating-characteristics analysis ( $P B, 3.68 \%$; $B M, 3.45 \%$ ). The high PB-CD11b+CX3CR1+ monocyte group was significantly associated with highintermediate and high risk NCCN-IPI group $(P=0.004)$. With a median follow-up of 27.7 months (range, 1.7-60.4 months), the low PB-CD11b+CX3CR1+ monocyte group showed significantly better overall survival (OS) than the high PB-CD11b+CX3CR1+ monocyte group (3-year, $92.3 \%$ vs. $51.2 \%$, respectively; $P<0.001$ ). In contrast, no significant difference was observed between the high and low BM-CD11b+CX3CR1 ${ }^{+}$ monocyte groups. Among patients with high-intermediate to high risk NCCN-IPI, the high PB-CD11b+CX3CR1+ monocyte group showed significantly worse OS than the low PB-CD11b ${ }^{+}$CX3CR1 ${ }^{+}$monocyte group (3-year, $29.3 \%$ vs. $80.2 \%$, respectively; $P=0.008)$. Taken together, $\mathrm{PB}-\mathrm{CD11} \mathrm{b}^{+} \mathrm{CX} 3 \mathrm{CR}^{+}$monocyte percentage correlates with the NCCN-IPI risk stratification, which enables identification of subgroups with extremely poor clinical outcomes. 


\section{INTRODUCTION}

Diffuse large B-cell lymphoma (DLBCL) is the most common histological subtype of aggressive non-Hodgkin's lymphomas in Korea [1]. Although DLBCL is classified as a single disease, it encompasses a wide variety of tumor biology and therefore results in different clinical behaviors, responses to therapy, and long-term outcomes [2]. Although the addition of rituximab to conventional therapy with cyclophosphamide, doxorubicin, vincristine, and prednisone (R-CHOP) has vastly improved clinical outcomes, a significant number of patients still experience treatment failure and eventually die [2]. Therefore, an improved risk stratification method and novel treatment approaches in groups at high risk of treatment failure are greatly needed.

Myeloid-lineage cells, including monocytes and their precursors, play central roles in cancer growth and progression by directly promoting angiogenesis $[3,4]$ and suppressing anti-tumor immunity [5-8]. Recently, interest in the prognostic role of myeloid-lineage cells in lymphoma has been increasing [7, 9-11]. Several investigators have reported that high circulating monocyte counts in combination with low lymphocyte counts have prognostic relevance in patients with DLBCL treated with R-CHOP, particularly high-risk DLBCL patients [10-12]. These findings suggest that specific subsets of monocytes may be responsible for cancer prognosis in DLBCL.

Among the various subsets of monocytes, cells expressing CD11b and CX3CR1 have been shown to promote angiogenesis through interactions with fractalkine (CX3CL1), the only ligand for CX3CR1, in mouse ischemic models [13]. $\mathrm{CD} 11 \mathrm{~b}^{+} \mathrm{CX} 3 \mathrm{CR} 1^{+}$monocytes are a subpopulation of $\mathrm{CD} 11 \mathrm{~b}^{+} \mathrm{Gr} 1^{+}$cells [14] that were shown to be myeloid-derived suppressor cells (MDSCs) in mouse tumor models [6]. Based on suppression of host anti-tumor immunity mediated by $\mathrm{CD} 11 \mathrm{~b}^{+} \mathrm{CX} 3 \mathrm{CR} 1^{+}$ monocytes in solid cancer models [15-17] and the upregulation of CX3CR1 receptor in various types of B-cell lymphoma including DLBCL [18], we hypothesized that $\mathrm{CD} 11 \mathrm{~b}^{+} \mathrm{CX} 3 \mathrm{CR} 1^{+}$monocytes might influence the prognosis of patients with DLBCL by promoting angiogenesis and immunosuppression within the tumor microenvironment. However, there has been no data on the prognostic relevance of $\mathrm{CD} 11 \mathrm{~b}^{+} \mathrm{CX} 3 \mathrm{CR} 1^{+}$monocytes in DLBCL as well as its relationship with clinical variables. Therefore, this prospective study investigated the prognostic significance of $\mathrm{CD} 11 \mathrm{~b}^{+} \mathrm{CX} 3 \mathrm{CR} 1^{+}$monocytes in peripheral blood (PB) and bone marrow (BM) on survival outcomes in newly-diagnosed DLBCL patients treated with R-CHOP immunochemotherapy.

\section{RESULTS}

\section{Patient cohort and characteristics}

Ninety-nine patients were screened for this study. Ten patients were excluded; nine patients did not meet the eligibility criteria, and one patient withdrew informed consent. A total of 89 patients were enrolled in the study, and their data were analyzed (Figure 1). The median age was 65 years at diagnosis (range, 19-88 years), and 52 patients $(58.4 \%)$ were male. Approximately half of the patients had advanced stage disease $(\mathrm{N}=45,50.6 \%)$ and elevated serum lactate dehydrogenase (LDH) levels (N $=46,51.7 \%$; Table 1). Fifty-two patients $(58.4 \%)$ were classified as non-germinal center B-cell like phenotype by immunohistochemistry [19]. Thirty-seven patients $(41.6 \%)$ were classified as high-intermediate or high risk based on the National Comprehensive Cancer NetworkInternational Prognostic Index (NCCN-IPI).

\section{Baseline PB- and BM-CD11b ${ }^{+}$CX3CR1 ${ }^{+}$ monocytes and survival outcomes}

Flow cytometric analyses of $\mathrm{PB}$ samples were successfully performed in all 89 patients. However, $\mathrm{BM}$ aspirate samples were analyzed in 88 patients, because one of the samples was not appropriate for analysis. $\mathrm{CD} 11 \mathrm{~b}^{+} \mathrm{CX} 3 \mathrm{CR} 1^{+}$monocytes were detectable in all $\mathrm{PB}$ and $\mathrm{BM}$ aspirate samples analyzed. The median percentages of $\mathrm{CD} 11 \mathrm{~b}^{+} \mathrm{CX} 3 \mathrm{CR} 1^{+}$monocytes were $3.31 \%$ (range, $0.21-21.66 \%$ ) in $\mathrm{PB}$ (PB-CD $11 \mathrm{~b}^{+} \mathrm{CX} 3 \mathrm{CR} 1^{+}$ monocytes) and $3.09 \%$ (range, $0.20-20.01 \%$ ) in $\mathrm{BM}$ $\left(\mathrm{BM}-\mathrm{CD} 11 \mathrm{~b}^{+} \mathrm{CX} 3 \mathrm{CR} 1^{+}\right.$monocytes). There was no association between the percentages of PB- and BM$\mathrm{CD} 11 \mathrm{~b}^{+} \mathrm{CX} 3 \mathrm{CR} 1^{+}$cells (Spearman correlation $=0.202, P$ $=0.132$; Figure 2).

Responses to R-CHOP therapy were evaluable in $85(95.5 \%)$ of the 89 patients (Supplementary Table 1). Among the evaluable patients, 70 patients (82.4\%) achieved a complete response (CR), which was significantly higher in patients with low or low-intermediate NCCN-IPI (46/49 [93.9\%] vs. 24/36 [66.7\%]; $P=0.001)$ and low PB-

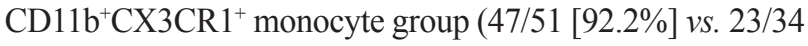
[66.7\%]; $P=0.004)$. During the median follow-up of 27.7 months (range, 1.7-60.4 months), 23 patients relapsed or progressed, and 18 patients died, including 1 patient with non-disease-related death. The estimated 3-year progressionfree survival (PFS) and overall survival (OS) rates were $68.7 \%$ (95\% confidence interval [CI], 56.9-80.5) and 76.8\% (95\% CI, 66.6-87.0), respectively. There were no significant differences in PFS $(P=0.193)$ or OS $(P=0.355)$ between the high and low $\mathrm{BM}-\mathrm{CD} 11 \mathrm{~b}{ }^{+} \mathrm{CX} 3 \mathrm{CR} 1^{+}$monocyte groups (Figure 3A, 3B). In contrast, the high PB-CD $11 \mathrm{~b}^{+} \mathrm{CX} 3 \mathrm{CR} 1^{+}$ monocyte group had more patients with relapse/progression $(16 / 37$ [43.2\%] vs. $7 / 52$ [13.5\%]) and death (13/37 [35.1\%] vs. $5 / 52[9.6 \%])$ than the low $\mathrm{PB}-\mathrm{CD} 11 \mathrm{~b}^{+} \mathrm{CX} 3 \mathrm{CR} 1^{+}$ monocyte group. Thus, high $\mathrm{PB}-\mathrm{CD} 11 \mathrm{~b}^{+} \mathrm{CX} 3 \mathrm{CR} 1^{+}$ monocyte group was significantly associated with worse PFS (3-year, $51.6 \%$ vs. $80.0 \% ; P<0.001$ ) and OS (3-year, $51.2 \%$ vs. $92.3 \% ; P<0.001$; Figure $3 \mathrm{C}, 3 \mathrm{D}$ ).

Univariate analyses of PFS and OS demonstrated that Eastern Cooperative Oncology Group (ECOG) performance status, extranodal involvement, risk stratification by NCCN- 
IPI (Supplementary Figure 1), and PB-CD $11 b^{+} \mathrm{CX} 3 \mathrm{CR} 1^{+}$ cell groups were significantly associated with PFS and OS (Table 1). Age $\geq 60$ years and the presence of B symptoms were also associated with increased death. However, high-intermediate to high risk NCCN-IPI was the only independent prognostic factor for shorter PFS (hazard ratio [HR], 3.67; 95\% CI, 1.40-9.62) and OS (HR, 6.25; 95\% CI, 1.76-22.18) in multivariate analysis (Table 1).

\section{Association between baseline PB- and BM- $\mathrm{CD} 11 \mathrm{~b}^{+} \mathrm{CX} 3 \mathrm{CR1}{ }^{+}$monocytes and other clinical variables}

We investigated the association of $\mathrm{CD} 11 \mathrm{~b}^{+} \mathrm{CX} 3 \mathrm{CR} 1^{+}$ monocyte percentages in $\mathrm{PB}$ or $\mathrm{BM}$ with other clinical variables. The high $\mathrm{PB}-\mathrm{CD} 11 \mathrm{~b}^{+} \mathrm{CX} 3 \mathrm{CR} 1^{+}$monocyte group was significantly associated with unfavorable clinical variables, including ECOG performance status $\geq 2(P=0.031)$, elevated serum LDH levels $(P=0.036)$, and extranodal involvement $(P=0.003)$, and also showed a trend towards older age $(P=0.099)$ (Table 2$)$. In particular, $\mathrm{PB}-\mathrm{CD} 11 \mathrm{~b}^{+} \mathrm{CX} 3 \mathrm{CR} 1^{+}$monocyte percentages were significantly associated with risk stratification by
NCCN-IPI $(P=0.004)$. However, BM-CD $11 b^{+}$CX3CR $1^{+}$ monocyte percentages were not significantly associated with any other clinical variables (Table 2).

Next, we classified the patients according to NCCNIPI risk and evaluated the impact of PB-CD $11 \mathrm{~b}^{+} \mathrm{CX} 3 \mathrm{CR} 1^{+}$ monocytes on survival outcomes in each subgroup. Thirtyseven patients $(41.6 \%)$ were classified as higher risk (highintermediate to high) subgroup and 52 patients (58.4\%) were classified as lower risk (low to low-intermediate) subgroups based on the NCCN-IPI. In the higher risk NCCN-IPI subgroup, patients with a high percentage of PB-CD $11 b^{+} \mathrm{CX} 3 \mathrm{CR}^{+}$monocytes had significantly worse PFS (3-year, 36.5\% vs. 59.4\%, respectively; $P=0.028$ ) and OS (3-year, $29.3 \%$ vs. $80.2 \%$, respectively; $P=0.008$ ) than those with a low percentage of $\mathrm{PB}-\mathrm{CD} 11 \mathrm{~b}^{+} \mathrm{CX} 3 \mathrm{CR} 1^{+}$ monocytes (Figure 4A, 4B). However, in the lower risk NCCN-IPI subgroup, the PB-CD $11 \mathrm{~b}^{+} \mathrm{CX} 3 \mathrm{CR} 1^{+}$monocyte percentages failed to predict PFS and OS (Figure 4C, 4D).

\section{DISCUSSION}

In this prospective cohort study involving 89 DLBCL patients, we investigated the impact of PB-

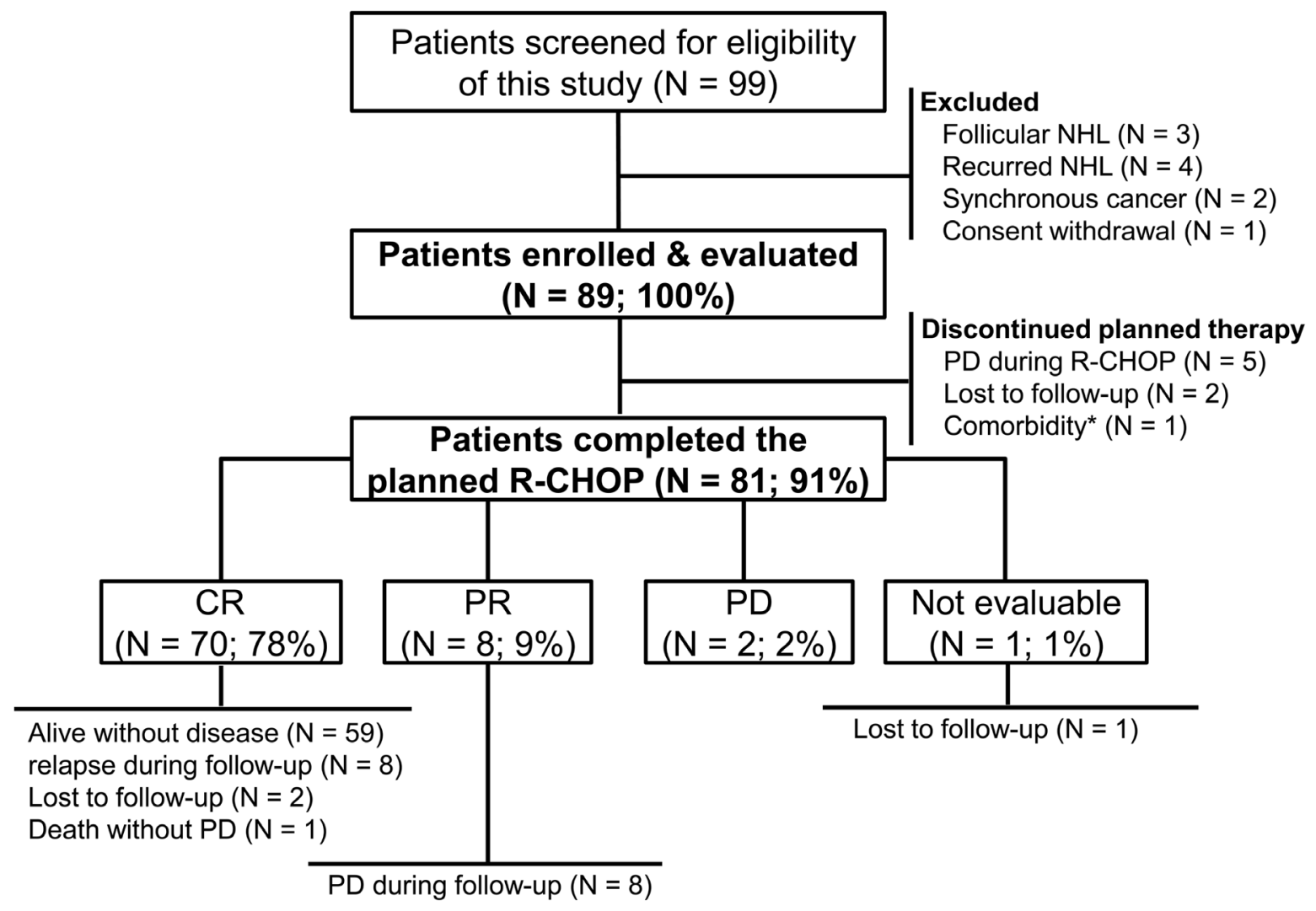

Figure 1: Flowchart of the study. NHL, non-Hodgkin lymphoma; R-CHOP, rituximab plus cyclophosphamide, doxorubicin, vincristine, and prednisone; $\mathrm{PD}$, progressive disease; $\mathrm{CR}$, complete response; PR, partial response. *Femur intertrochanteric fracture following a fall by a 79-year-old woman. 
Table 1: Clinical characteristics and the association with PFS and OS

\begin{tabular}{|c|c|c|c|c|c|c|c|c|c|}
\hline \multirow{3}{*}{ Variables } & \multirow{3}{*}{ No. $(\%)$} & \multicolumn{4}{|c|}{ PFS } & \multicolumn{4}{|c|}{ os } \\
\hline & & \multicolumn{2}{|c|}{ Univariate analysis } & \multicolumn{2}{|c|}{ Multivariate analysis } & \multicolumn{2}{|c|}{ Univariate analysis } & \multicolumn{2}{|c|}{ Multivariate analysis } \\
\hline & & 3 -year $(\%, 95 \%$ CI $)$ & $P$ & HR $(95 \%$ CI) & $P$ & 3 -year $(\%, 95 \%$ CI $)$ & $P$ & HR (95\% CI) & $P$ \\
\hline $\begin{array}{l}\text { Age (years) } \\
\quad<60 \\
\geq 60\end{array}$ & $\begin{array}{l}38(42.7) \\
51(57.3)\end{array}$ & $\begin{array}{l}75.3(57.7-92.9) \\
63.5(47.8-79.2)\end{array}$ & 0.218 & & & $\begin{array}{c}89.5(78.3-100.0) \\
66.7(51.4-82.0)\end{array}$ & 0.045 & & \\
\hline $\begin{array}{l}\text { Sex } \\
\quad \text { Male } \\
\text { Female }\end{array}$ & $\begin{array}{l}52(58.4) \\
37(41.6)\end{array}$ & $\begin{array}{l}71.4(56.7-86.1) \\
65.7(47.3-84.1)\end{array}$ & 0.488 & & & $\begin{array}{l}81.4(69.4-93.4) \\
69.2(51.0-87.4)\end{array}$ & 0.551 & & \\
\hline $\begin{array}{l}\text { Ann Arbor stage } \\
\text { I to II } \\
\text { III to IV }\end{array}$ & $\begin{array}{l}44(49.4) \\
45(50.6)\end{array}$ & $\begin{array}{l}74.5(58.8-90.2) \\
64.1(44.7-79.5)\end{array}$ & 0.280 & & & $\begin{array}{l}86.1(74.5-97.7) \\
66.6(50.1-83.1)\end{array}$ & 0.083 & & \\
\hline $\begin{array}{l}\text { Performance status } \\
\text { ECOG } 0 / 1 \\
\text { ECOG } \geq 2\end{array}$ & $\begin{array}{l}74(83.1) \\
15(16.9)\end{array}$ & $\begin{array}{c}78.8(67.2-90.4) \\
28.0(2.9-53.1)\end{array}$ & $<0.001$ & & & $\begin{array}{l}86.5(77.1-95.9) \\
37.3(11.2-63.4)\end{array}$ & $<0.001$ & & \\
\hline $\begin{array}{l}\text { Serum LDH level } \\
\text { Normal } \\
\text { Elevated }\end{array}$ & $\begin{array}{l}43(48.3) \\
46(51.7)\end{array}$ & $\begin{array}{l}69.1(53.0-85.2) \\
69.3(52.6-86.0)\end{array}$ & 0.886 & & & $\begin{array}{l}78.1(64.6-91.6) \\
75.8(60.7-90.9)\end{array}$ & 0.766 & & \\
\hline $\begin{array}{l}\text { B symptoms } \\
\text { Absence } \\
\text { Presence }\end{array}$ & $\begin{array}{l}59(66.3) \\
30(33.7)\end{array}$ & $\begin{array}{l}74.7(61.6-87.8) \\
57.6(35.8-79.4)\end{array}$ & 0.202 & & & $\begin{array}{l}85.0(74.6-95.4) \\
60.4(39.6-81.2)\end{array}$ & 0.026 & & \\
\hline $\begin{array}{l}\text { Bulky disease } \\
\text { No } \\
\text { Yes }\end{array}$ & $\begin{array}{c}80(89.9) \\
9(10.1)\end{array}$ & $\begin{array}{c}68.2(55.7-80.7) \\
71.1(35.8-100.0)\end{array}$ & 0.890 & & & $\begin{array}{c}77.3(66.7-87.9) \\
71.1(35.8-100.0)\end{array}$ & 0.818 & & \\
\hline $\begin{array}{l}\text { Extranodal involvement } \\
\text { No } \\
\text { Yes }\end{array}$ & $\begin{array}{l}34(38.2) \\
55(61.8)\end{array}$ & $\begin{array}{l}86.1(73.4-98.8) \\
58.5(42.4-74.6)\end{array}$ & 0.031 & & & $\begin{array}{c}88.7(76.4-100.0) \\
69.7(55.6-83.8)\end{array}$ & 0.045 & & \\
\hline $\begin{array}{l}\text { Cell of origin } \\
\text { GCB phenotype } \\
\text { Non-GCB phenotype } \\
\text { Unknown }\end{array}$ & $\begin{array}{l}35(39.3) \\
52(58.4) \\
2(2.3)\end{array}$ & $\begin{array}{c}73.2(56.5-89.9) \\
65.2(48.9-81.5) \\
100.0\end{array}$ & 0.482 & & & $\begin{array}{c}75.6(59.7-91.5) \\
77.4(64.1-90.7) \\
100.0\end{array}$ & 0.900 & & \\
\hline $\begin{array}{l}\text { NCCN-IPI } \\
\text { Low/low-intermediate } \\
\text { High-intermediate/high }\end{array}$ & $\begin{array}{l}52(58.4) \\
37(41.6)\end{array}$ & $\begin{array}{l}83.6(70.5-96.7) \\
47.3(27.7-66.9)\end{array}$ & $<0.001$ & $\begin{array}{c}1 \\
3.67(1.40-9.62)\end{array}$ & 0.008 & $\begin{array}{c}95.1(88.4-100.0) \\
51.3(32.1-70.5)\end{array}$ & $<0.001$ & $\begin{array}{c}1 \\
6.25(1.76-22.18)\end{array}$ & 0.005 \\
\hline $\begin{array}{l}\text { PB-CD } 11 b^{+} \text {CX3CR } 1^{+} \text {cells } \\
\text { Low } \\
\text { High }\end{array}$ & $\begin{array}{l}52(58.4) \\
37(41.6)\end{array}$ & $\begin{array}{l}80.0(65.7-94.3) \\
51.6(42.1-61.1)\end{array}$ & $<0.001$ & & & $\begin{array}{c}92.3(83.9-100.0) \\
51.2(31.2-71.2)\end{array}$ & $<0.001$ & & \\
\hline $\begin{array}{l}\text { BM-CD11b+CX3CR1 } 1^{+} \text {cells } \\
\text { Low } \\
\text { High }\end{array}$ & $\begin{array}{l}50(56.8) \\
38(43.2)\end{array}$ & $\begin{array}{l}75.3(59.4-91.2) \\
60.1(42.7-77.5)\end{array}$ & 0.193 & & & $\begin{array}{l}81.3(68.6-94.0) \\
71.3(55.0-87.6)\end{array}$ & 0.355 & & \\
\hline $\begin{array}{l}\text { PB-/BM-CD } 11 \mathrm{~b}^{+} \mathrm{CX} 3 \mathrm{CR} 1^{+} \text {cells ratio } \\
\quad \leq 1.77 \\
>1.77\end{array}$ & $\begin{array}{l}61(69.3) \\
27(30.7)\end{array}$ & $\begin{array}{l}70.7(56.0-85.4) \\
63.6(44.2-83.0)\end{array}$ & 0.118 & & & $\begin{array}{l}78.4(66.2-90.6) \\
72.9(54.3-91.5)\end{array}$ & 0.811 & & \\
\hline
\end{tabular}

Abbreviations: PFS, progression-free survival; OS, overall survival; CI, confidence interval; ECOG, Eastern Cooperative Oncology Group; LDH, lactate dehydrogenase; GCB, germinal center B-cell like; NCCN-IPI, National Comprehensive Cancer Network-International Prognostic Index (NCCN-IPI); PB, peripheral blood; BM, bone marrow.

and $\mathrm{BM}-\mathrm{CD} 11 \mathrm{~b}^{+} \mathrm{CX} 3 \mathrm{CR} 1^{+}$monocytes on survival outcomes in patients with newly diagnosed DLBCL treated with R-CHOP immunochemotherapy. Our study demonstrated that the patients with high percentage of $\mathrm{PB}$ $\mathrm{CD} 11 \mathrm{~b}^{+} \mathrm{CX} 3 \mathrm{CR}^{+}$monocytes were significantly associated with disease progression and death, compared to those with low percentage of PB-CD $11 \mathrm{~b}^{+} \mathrm{CX} 3 \mathrm{CR} 1^{+}$monocytes. A high percentage of $\mathrm{PB}-\mathrm{CD} 11 \mathrm{~b}^{+} \mathrm{CX} 3 \mathrm{CR} 1^{+}$monocytes was also strongly associated with several poor prognostic variables, including poor ECOG performance status, elevated serum LDH levels, extranodal involvement, and higher NCCN-IPI risk. Furthermore, the baseline percentage of $\mathrm{PB}-\mathrm{CD} 11 \mathrm{~b}^{+} \mathrm{CX} 3 \mathrm{CR} 1^{+}$monocytes allowed us to identify the subgroup with very poor outcomes among patients with higher risk NCCN-IPI.

Prior to the present study, there were no data regarding the prognostic value of $\mathrm{CD} 11 \mathrm{~b}^{+} \mathrm{CX} 3 \mathrm{CR} 1^{+}$ monocytes in DLBCL. As we hypothesized, a high percentage of $\mathrm{PB}-\mathrm{CD} 11 \mathrm{~b}^{+} \mathrm{CX} 3 \mathrm{CR} 1^{+}$monocytes was clearly associated with disease progression and death. The significant role of $\mathrm{PB}-\mathrm{CD} 11 \mathrm{~b}^{+} \mathrm{CX} 3 \mathrm{CR} 1^{+}$monocytes on PFS and OS in patients with newly diagnosed DLBCL might be attributed to the pro-angiogenic capacity of these cells. In the tumor microenvironment, myeloid-lineage cells play important roles in promoting tumor angiogenesis [3, 4]. Yang et al. [4] reported that myeloid-lineage cells expressing CD11b and Gr1 directly contributed to tumor angiogenesis. However, $\mathrm{CD}_{11} \mathrm{~b}^{+} \mathrm{Gr} 1^{+}$cells are heterogeneous, containing various subpopulations, such

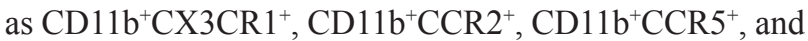

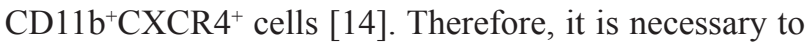
define the specific subset that enhances tumor progression. Recently, CD $11 \mathrm{~b}^{+} \mathrm{CX} 3 \mathrm{CR} 1^{+}$monocytes have been found to strongly promote endothelial proliferation in human 
umbilical vein endothelial cell culture through CX3CR1/ fractalkine interaction [13]. Although the pro-angiogenic effect of $\mathrm{CD} 11 \mathrm{~b}^{+} \mathrm{CX} 3 \mathrm{CR} 1^{+}$monocytes has not been well described in human cancer types, these findings suggest that $\mathrm{CD} 11 \mathrm{~b}^{+} \mathrm{CX} 3 \mathrm{CR} 1^{+}$monocytes might promote tumor growth and progression by enhancing angiogenesis in DLBCL.

In addition to their pro-angiogenic capacity, PB$\mathrm{CD}_{11} \mathrm{~b}^{+} \mathrm{CX} 3 \mathrm{CR}^{+}$monocytes can suppress host antitumor immune responses. In a mouse model of ovarian cancer, CX3CR1-expressing myeloid cells promoted immunosuppression through inhibition of T-cell activity by IL-10 $[15,20]$, suggesting that $\mathrm{CD} 11 \mathrm{~b}^{+} \mathrm{CX} 3 \mathrm{CR} 1^{+}$ cells function as MDSCs. In this study, we identified

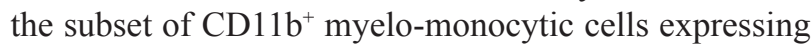
CX3CR1 in the PB and BM of DLBCL patients. Since these cells were detected in both mice and humans [14], we postulated that they promote the immunosuppressive tumor microenvironment in DLBCL patients through immunosuppressive mechanisms similar to those observed in mice. Therefore, although previous studies have suggested the immunosuppressive role of $\mathrm{CD} 11 \mathrm{~b}^{+} \mathrm{CX} 3 \mathrm{CR} 1^{+}$monocytes in the tumor microenvironment, the mechanism of these cells in DLBCL needs to be further clarified.

In contrast to $\mathrm{PB}-\mathrm{CD} 11 \mathrm{~b}^{+} \mathrm{CX} 3 \mathrm{CR} 1^{+}$monocytes, $\mathrm{BM}-\mathrm{CD} 11 \mathrm{~b}{ }^{+} \mathrm{CX} 3 \mathrm{CR} 1^{+}$monocytes were not associated with prognosis of DLBCL patients. It is unclear why only $\mathrm{PB}-\mathrm{CD} 11 \mathrm{~b}^{+} \mathrm{CX} 3 \mathrm{CR}^{+}$monocytes affect the prognosis of DLBCL patients. Considering that BM-derived myeloidlineage cells are generally under steady state conditions, our findings suggest that additional stimulants may be required to mobilize these cells from the $\mathrm{BM}$ into the $\mathrm{PB}$ [21]. Fractalkine, a unique member of the CX3C family, is a transmembrane protein that can be cleaved into a soluble form with potent chemoattractant properties for monocytes [22, 23]. Truman L et al. [24] reported that apoptotic lymphoma cells released fractalkine and recruited CX3CR1-expressing monocytes to the germinal center in a mouse model of lymphoma. Therefore, we

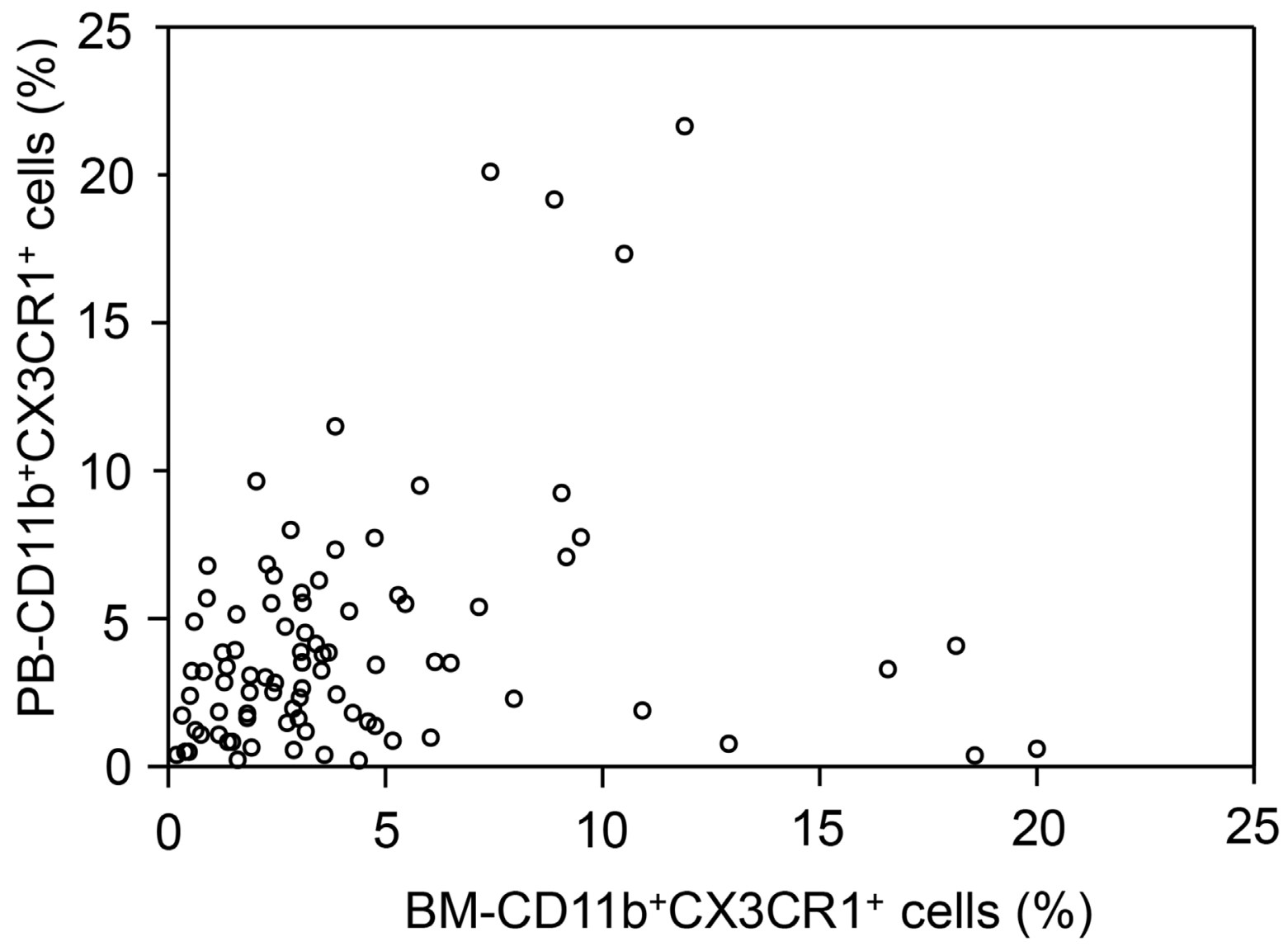

Figure 2: Scatter plot of the percentages of bone marrow (BM)-CD11 $b^{+} C_{X X 3 C 1^{+}}$versus peripheral (PB)$\mathrm{CD}_{11} \mathrm{~b}^{+}$CX3CR1 ${ }^{+}$monocytes in each patient. A dot was not marked on this plot for one patient because $\mathrm{BM}-\mathrm{CD} 11 \mathrm{~b}^{+} \mathrm{CX} 3 \mathrm{CR} 1^{+}$ monocyte percentage was not available. There was no association between the percentages of PB- and BM-CD $11 \mathrm{~b}^{+} \mathrm{CX} 3 \mathrm{CR} 1^{+}$monocytes (Spearman correlation coefficient $=0.202 ; P=0.132$ ). 
postulate that tumor-derived fractalkine release stimulates mobilization of $\mathrm{CD} 11 \mathrm{~b}^{+} \mathrm{CX} 3 \mathrm{CR} 1^{+}$monocytes through fractalkine/CX3CR1 interaction, and these mobilized $\mathrm{CD} 11 \mathrm{~b}^{+} \mathrm{CX} 3 \mathrm{CR} 1^{+}$monocytes in the $\mathrm{PB}$ have angiogenic and/or immunosuppressive activity in the tumor microenvironment.

Our study also demonstrated that PB$\mathrm{CD}_{11} \mathrm{~b}^{+} \mathrm{CX} 3 \mathrm{CR} 1^{+}$monocytes were strongly correlated with the risk stratification by NCCN-IPI, demonstrating the association of $\mathrm{PB}-\mathrm{CD} 11 \mathrm{~b}^{+} \mathrm{CX} 3 \mathrm{CR} 1^{+}$monocytes with clinical variables in DLBCL. NCCN-IPI is a new prognostic tool in the era of rituximab-based therapy, which incorporates a refined categorization of age, serum LDH level, and disease involvement at specific extranodal sites (i.e., BM, central nervous system, liver, gastrointestinal tract, or lung) [25]. With regards to age and serum LDH level, NCCN-IPI shows more detailed values; therefore, it is able to discriminate high risk groups better than the original IPI [25]. In addition to the enhanced capacity of NCCN-IPI for predicting prognosis
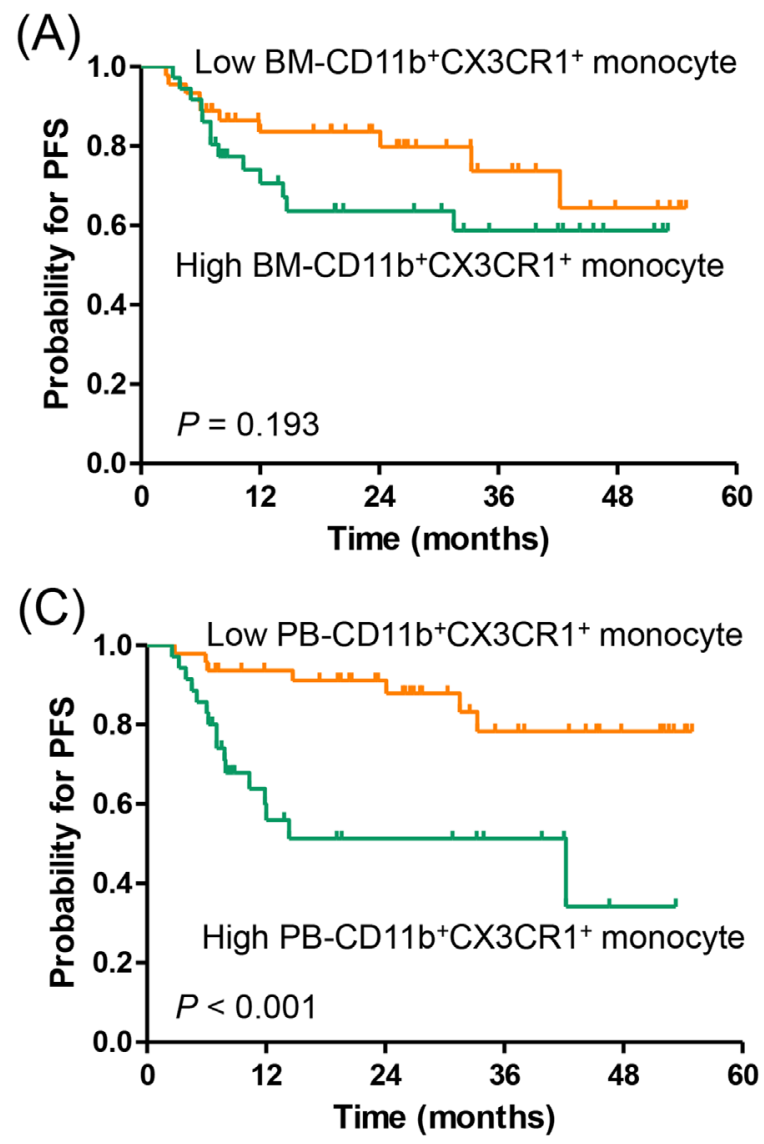

in DLBCL, the results of our study revealed that PB$\mathrm{CD}_{11} \mathrm{~b}^{+} \mathrm{CX} 3 \mathrm{CR}^{+}$monocyte level might be used to further discriminate the risk of disease progression and death in higher risk NCCN-IPI subgroup. Thus, our results will be useful for discriminating patients at high risk for disease progression and death. Furthermore, these findings have therapeutic implications. For lower risk NCCN-IPI subgroup, traditional R-CHOP chemotherapy shows excellent outcomes regardless of PB-CD $11 \mathrm{~b}^{+} \mathrm{CX} 3 \mathrm{CR} 1^{+}$ monocyte status. In contrast, patients in the higher risk NCCN-IPI subgroup with high $\mathrm{PB}-\mathrm{CD} 11 \mathrm{~b}^{+} \mathrm{CX} 3 \mathrm{CR} 1^{+}$ monocyte percentages showed very poor OS (3-year, $29 \%$ ). Interestingly, the lower survival rate observed in this study may be improved by adding novel drugs that inhibit fractalkine/CX3CR1 signaling. A novel small-molecule CX3CR1 antagonist has shown preclinical evidence of activity by interfering with progression and metastasis in a breast cancer model [26]. Thus, clinical application of new therapeutic agents that target the fractalkine/CX3CR1
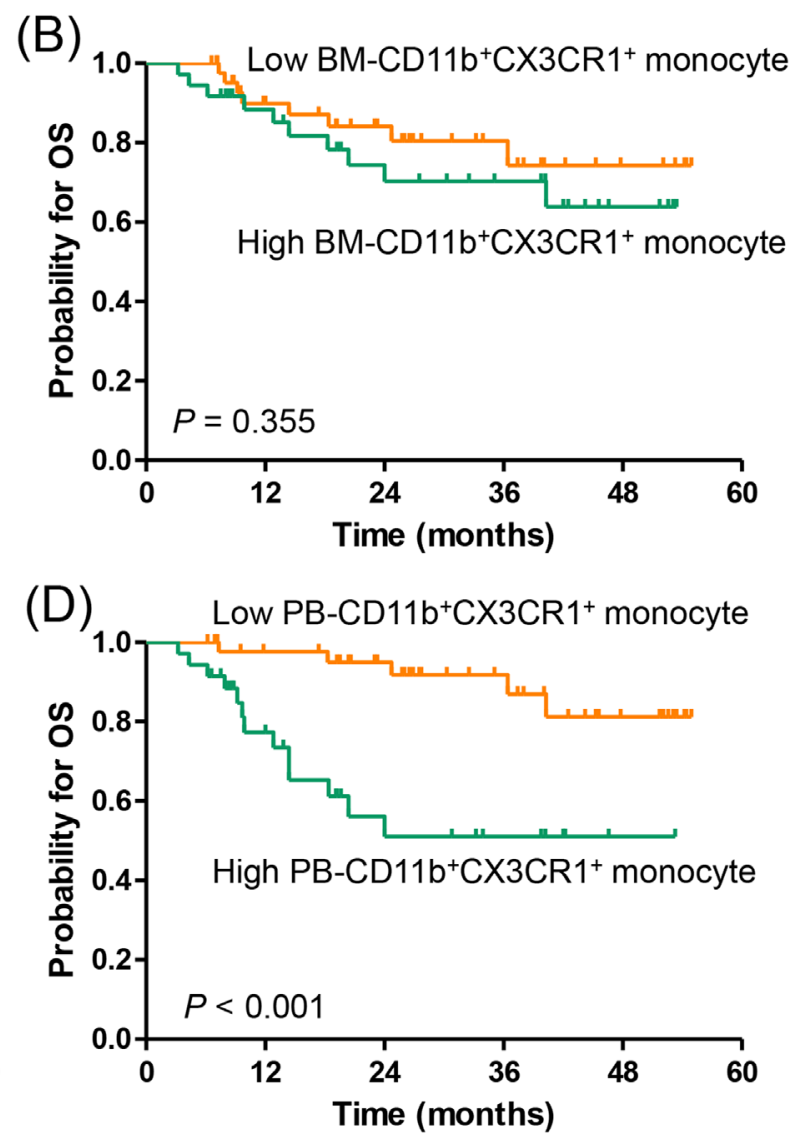

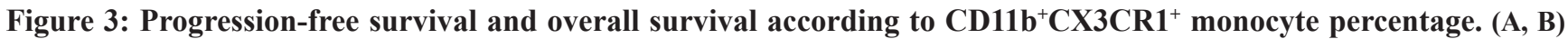
bone marrow aspirate and $(\mathbf{C}, \mathbf{D})$ peripheral blood samples.PFS, progression-free survival; OS, overall survival; BM, bone marrow; PB, peripheral blood. 
Table 2: The association between baseline $\mathrm{CD}_{11}{ }^{+} \mathrm{CX} 3 \mathrm{CR} 1^{+}$cells and clinical variables

\begin{tabular}{|c|c|c|c|c|c|c|c|c|}
\hline \multirow[b]{2}{*}{ Variables } & \multicolumn{4}{|c|}{ PB-CD11b $^{+}$CX3CR1 ${ }^{+}$cells $(N=89)$} & \multicolumn{4}{|c|}{ BM-CD11b $^{+} \mathrm{CX} 3 \mathrm{CR} 1^{+}$cells $(\mathrm{N}=88)$} \\
\hline & No. $(\%)$ & $\begin{array}{c}\operatorname{Low}(\mathbf{N}= \\
52, \%)\end{array}$ & $\begin{array}{c}\text { High }(\mathbf{N}= \\
37, \%)\end{array}$ & $\boldsymbol{P}$ & No. $(\%)$ & $\begin{array}{c}\text { Low }(\mathrm{N}= \\
50, \%)\end{array}$ & $\begin{array}{c}\text { High }(\mathrm{N}= \\
\mathbf{3 8}, \%)\end{array}$ & $\boldsymbol{P}$ \\
\hline $\begin{array}{l}\text { Age (years) } \\
\quad<60 \\
\quad \geq 60\end{array}$ & $\begin{array}{l}38(42.7) \\
51(57.3)\end{array}$ & $\begin{array}{l}26(50.0) \\
26(50.0)\end{array}$ & $\begin{array}{l}12(32.4) \\
25(67.6)\end{array}$ & 0.099 & $\begin{array}{l}38(43.2) \\
50(56.8)\end{array}$ & $\begin{array}{l}23(46.0) \\
27(54.0)\end{array}$ & $\begin{array}{l}15(39.5) \\
23(60.5)\end{array}$ & 0.540 \\
\hline $\begin{array}{l}\text { Sex } \\
\quad \text { Male } \\
\text { Female }\end{array}$ & $\begin{array}{l}52(58.4) \\
37(41.6)\end{array}$ & $\begin{array}{l}32(61.5) \\
20(38.5)\end{array}$ & $\begin{array}{l}20(54.1) \\
17(45.9)\end{array}$ & 0.480 & $\begin{array}{l}51(58.0) \\
37(42.0)\end{array}$ & $\begin{array}{l}27(54.0) \\
23(46.0)\end{array}$ & $\begin{array}{l}24(63.2) \\
14(36.8)\end{array}$ & 0.389 \\
\hline $\begin{array}{l}\text { Ann Arbor stage } \\
\text { I to II } \\
\text { III to IV }\end{array}$ & $\begin{array}{l}44(49.4) \\
45(50.6)\end{array}$ & $\begin{array}{l}28(53.8) \\
24(46.2)\end{array}$ & $\begin{array}{l}16(43.2) \\
21(56.8)\end{array}$ & 0.324 & $\begin{array}{l}44(50.0) \\
44(50.0)\end{array}$ & $\begin{array}{l}25(50.0) \\
25(50.0)\end{array}$ & $\begin{array}{l}19(50.0) \\
19(50.0)\end{array}$ & 1.000 \\
\hline $\begin{array}{l}\text { Performance status } \\
\qquad \begin{array}{l}\text { ECOG } 0 / 1 \\
\text { ECOG } \geq 2\end{array}\end{array}$ & $\begin{array}{l}74(83.1) \\
15(16.9)\end{array}$ & $\begin{array}{c}47(90.4) \\
5(9.6)\end{array}$ & $\begin{array}{l}27(73.0) \\
10(27.0)\end{array}$ & 0.031 & $\begin{array}{l}73(83.0) \\
15(17.0)\end{array}$ & $\begin{array}{c}44(88.0) \\
6(12.0)\end{array}$ & $\begin{array}{c}29(76.3) \\
9(23.7)\end{array}$ & 0.149 \\
\hline $\begin{array}{l}\text { Serum LDH level } \\
\text { Normal } \\
\text { Elevated }\end{array}$ & $\begin{array}{l}43(48.3) \\
46(51.7)\end{array}$ & $\begin{array}{l}30(57.7) \\
22(42.3)\end{array}$ & $\begin{array}{l}13(35.1) \\
24(64.9)\end{array}$ & 0.036 & $\begin{array}{l}43(48.9) \\
45(51.1)\end{array}$ & $\begin{array}{l}25(50.0) \\
25(50.0)\end{array}$ & $\begin{array}{l}18(47.4) \\
20(52.6)\end{array}$ & 0.807 \\
\hline $\begin{array}{l}\text { B symptoms } \\
\text { Absence } \\
\text { Presence }\end{array}$ & $\begin{array}{l}59(66.3) \\
30(33.7)\end{array}$ & $\begin{array}{l}36(69.2) \\
16(30.8)\end{array}$ & $\begin{array}{l}23(62.2) \\
14(37.8)\end{array}$ & 0.487 & $\begin{array}{l}58(65.9) \\
30(34.1)\end{array}$ & $\begin{array}{l}36(72.0) \\
14(28.0)\end{array}$ & $\begin{array}{l}22(57.9) \\
16(42.1)\end{array}$ & 0.167 \\
\hline $\begin{array}{l}\text { Bulky disease } \\
\text { No } \\
\text { Yes }\end{array}$ & $\begin{array}{c}80(89.9) \\
9(10.1)\end{array}$ & $\begin{array}{c}47(90.4) \\
5(9.6)\end{array}$ & $\begin{array}{c}33(89.2) \\
4(10.8)\end{array}$ & 0.854 & $\begin{array}{c}79(89.8) \\
9(10.2)\end{array}$ & $\begin{array}{c}46(92.0) \\
4(8.0)\end{array}$ & $\begin{array}{c}33(86.8) \\
5(13.2)\end{array}$ & 0.429 \\
\hline $\begin{array}{l}\text { Extranodal } \\
\text { involvement } \\
\text { No } \\
\text { Yes }\end{array}$ & $\begin{array}{l}34(38.2) \\
55(61.8)\end{array}$ & $\begin{array}{l}25(48.1) \\
27(51.9)\end{array}$ & $\begin{array}{c}9(24.3) \\
28(75.7)\end{array}$ & 0.023 & $\begin{array}{l}34(38.2) \\
54(61.8)\end{array}$ & $\begin{array}{l}22(44.0) \\
28(56.0)\end{array}$ & $\begin{array}{l}12(31.6) \\
26(68.4)\end{array}$ & 0.236 \\
\hline $\begin{array}{l}\text { Cell of origin } \\
\text { GCB } \\
\text { Non-GCB }\end{array}$ & $\begin{array}{l}87(100.0) \\
35(40.2) \\
52(59.8)\end{array}$ & $\begin{array}{l}22(43.1) \\
29(56.9)\end{array}$ & $\begin{array}{l}13(36.1) \\
23(63.9)\end{array}$ & 0.510 & $\begin{array}{c}86(100.0) \\
35(40.7) \\
51(59.3)\end{array}$ & $\begin{array}{l}20(41.7) \\
28(58.3)\end{array}$ & $\begin{array}{l}15(39.5) \\
23(60.5)\end{array}$ & 0.837 \\
\hline $\begin{array}{l}\text { NCCN-IPI } \\
\text { Low/low- } \\
\text { intermediate } \\
\text { High-intermediate/ } \\
\text { high }\end{array}$ & $\begin{array}{l}52(58.4) \\
37(41.6)\end{array}$ & $\begin{array}{l}37(71.2) \\
15(28.8)\end{array}$ & $\begin{array}{l}15(40.5) \\
22(59.5)\end{array}$ & 0.004 & $\begin{array}{l}52(59.1) \\
36(40.9)\end{array}$ & $\begin{array}{l}32(64.0) \\
18(36.0)\end{array}$ & $\begin{array}{l}20(52.6) \\
18(47.4)\end{array}$ & 0.283 \\
\hline
\end{tabular}

Abbreviations: BM, bone marrow; ECOG, Eastern Cooperative Oncology Group; LDH, lactate dehydrogenase; GCB, germinal center B-cell like; NCCN-IPI, National Comprehensive Cancer Network-International Prognostic Index.

axis could be applied in the high risk DLBCL patients with high percentage of $\mathrm{PB}-\mathrm{CD} 11 \mathrm{~b}^{+} \mathrm{CX} 3 \mathrm{CR} 1^{+}$monocytes.

A potential limitation of our study is the relatively small number of patients with short follow-up period (approximately 2.3 years). Indeed, only approximately $20 \%$ of patients died, reflecting the limitation of an arbitrary cutoff rather than the biological cutoff that reflects the different clinical outcomes between the high and low $\mathrm{CD} 11 \mathrm{~b}^{+} \mathrm{CX} 3 \mathrm{CR} 1^{+}$monocyte groups. Furthermore, although our subgroup analysis provided some useful information, the results of the subgroup analysis might be underpowered. Future studies with larger populations are required to bolster our results.

Taken together, our study establishes the significant association of $\mathrm{PB}-\mathrm{CD} 11 \mathrm{~b}^{+} \mathrm{CX} 3 \mathrm{CR} 1^{+}$monocytes with risk stratification with the NCCN-IPI, and also identifies 
(A)

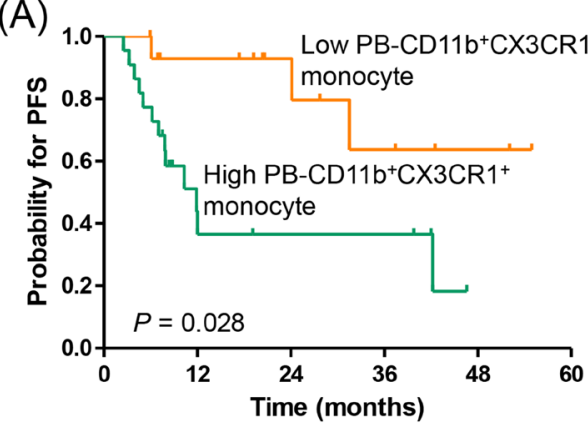

(C)

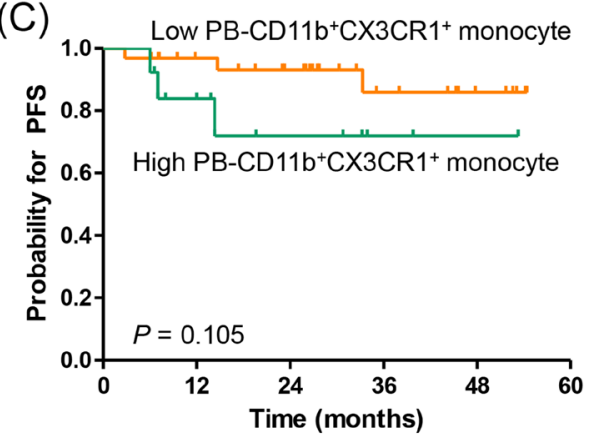

(B)

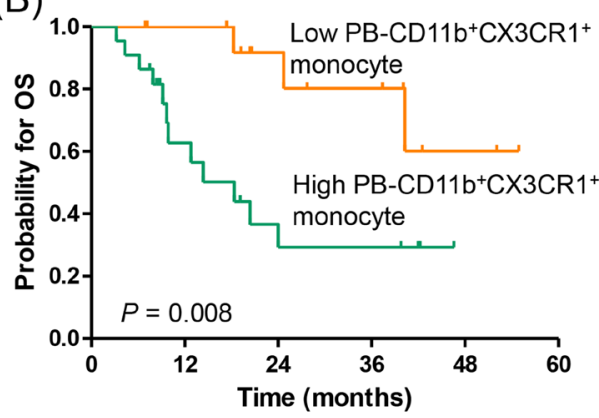

(D)

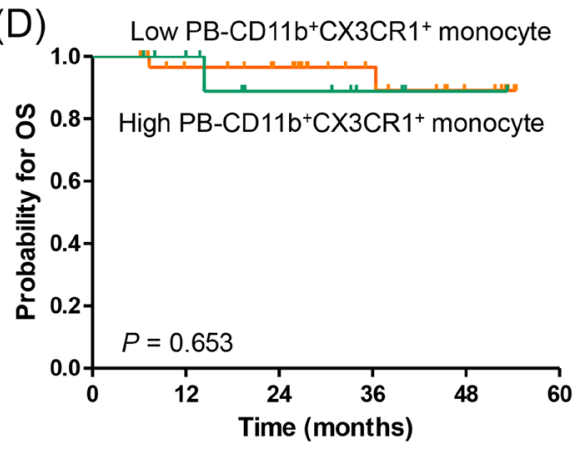

Figure 4: Subgroup analysis for the association of PB-CD11 ${ }^{+} \mathrm{CX} 3 \mathrm{CR} 1^{+}$monocytes with progression-free survival and overall survival based on NCCN-IPI risk. (A, B) high-intermediate to high risk NCCN-IPI (N = 37), (C, D) low to low-intermediate risk NCCN-IPI (N = 52). PFS, progression-free survival; OS, overall survival; BM, bone marrow; PB, peripheral blood.
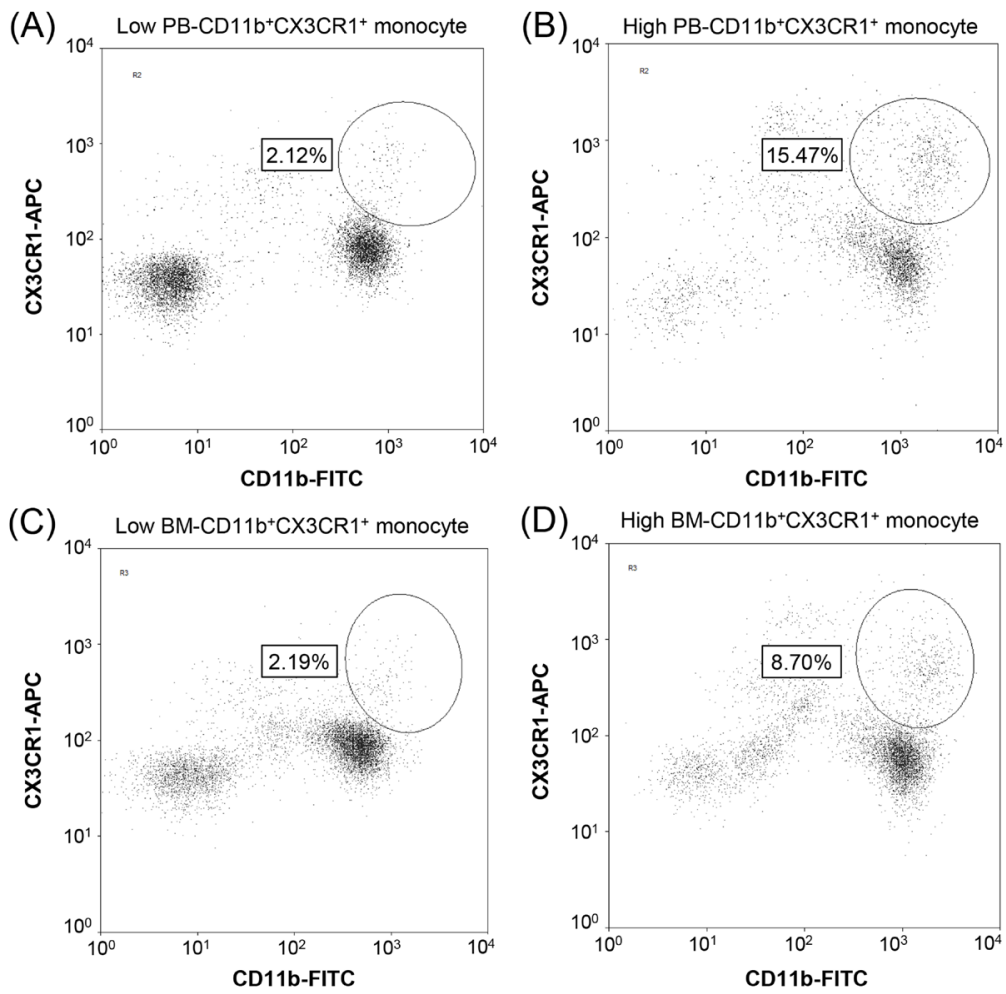

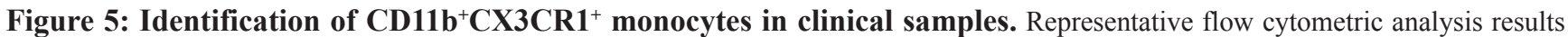
showing the percentages of $\mathrm{CD} 11 \mathrm{~b}^{+} \mathrm{CX} 3 \mathrm{CR} 1^{+}$monocytes in peripheral blood $(\mathbf{A}, \mathbf{B})$ and bone marrow $(\mathbf{C}, \mathbf{D})$ aspirate samples, which were measured as the percentage of $\mathrm{CD} 11 \mathrm{~b}^{+} \mathrm{CX} 3 \mathrm{CR} 1^{+}$monocytes among total mononuclear cells (> 50,000 cells). Patients were categorized into low $(\mathrm{A}, \mathrm{C})$ or high $(\mathrm{B}, \mathrm{D}) \mathrm{PB}$ - and $\mathrm{BM}-\mathrm{CD} 11 \mathrm{~b}^{+} \mathrm{CX} 3 \mathrm{CR} 1^{+}$monocyte groups. 
a subgroup with extremely poor clinical outcomes. This study also suggests the possibility of PB-CD $11 \mathrm{~b}^{+} \mathrm{CX} 3 \mathrm{CR} 1^{+}$ monocytes as a potential therapeutic target in higher risk subgroup of DLBCL.

\section{MATERIALS AND METHODS}

\section{Study design and eligibility criteria}

From May 2011 to August 2015, this multicenter, prospective, pilot study included patients with newly diagnosed, biopsy-proven, CD20-positive DLBCL. Other eligibility criteria included no prior history of chemotherapy or radiotherapy and adequate organ function to receive chemotherapy with curative intent. Patients were excluded if they had secondary transformed DLBCL, symptomatic central nervous system involvement, or other concurrent cancers requiring treatments. Patients suffering from severe infection, liver cirrhosis, or psychiatric disorder were also excluded. This study was approved by the Institutional Review Board at each institution. All patients provided written informed consent.

\section{Evaluation and treatment}

Patients were staged according to the Ann Arbor staging system. Baseline staging evaluation included a physical examination, complete blood count, serum biochemistry with LDH, computed tomographic (CT) scanning of the chest and abdomen-pelvis, CT scanning or magnetic resonance imaging of other involved lesions, fluorodeoxyglucose positron emission tomography, and bilateral BM aspiration and trephine biopsy. Predefined treatment consisted of six to eight cycles of standard R-CHOP immunochemotherapy (rituximab $375 \mathrm{mg}$ / $\mathrm{m}^{2}$, cyclophosphamide $750 \mathrm{mg} / \mathrm{m}^{2}$, doxorubicin $50 \mathrm{mg} /$ $\mathrm{m}^{2}$, and vincristine $1.4 \mathrm{mg} / \mathrm{m}^{2}$ intravenously one day 1 , and prednisone $100 \mathrm{mg}$ orally for 5 days) every 21 days. Prophylactic granulocyte-colony stimulating factor was recommended but was not mandated. Dose modification with respect to adverse effects in the preceding cycle was performed at the discretion of treating physician. The NCCN-IPI was determined for prognosis [25]. Responses to treatment were assessed by modified response criteria [27].

\section{Measurements of baseline $\mathrm{CD} 11 \mathrm{~b}^{+} \mathrm{CX3CR1^{+ }}$ monocytes in $\mathrm{PB}$ and $\mathrm{BM}$ aspirate samples}

The percentage of $\mathrm{CD} 11 \mathrm{~b}^{+} \mathrm{CX} 3 \mathrm{CR} 1^{+}$cells among the total mononuclear cells (MNCs) was measured by flow cytometric analysis in fresh $\mathrm{PB}$ and $\mathrm{BM}$ aspirate samples before administration of R-CHOP therapy. PBand $\mathrm{BM}-\mathrm{CD} 11 \mathrm{~b}^{+} \mathrm{CX} 3 \mathrm{CR} 1^{+}$cells were measured by the same procedure. Briefly, $2 \mathrm{~mL}$ of $\mathrm{PB}$ or $\mathrm{BM}$ aspirates was added to $20 \mathrm{~mL}$ of RBC lysis buffer $(1 \times$, Biolegend,
San Diego, CA), and then the cells were stained with APC-conjugated anti-human CX3CR1 antibody (Ab; Biolegend, San Diego, CA; catalog number 2A9-1), and FITC-conjugated anti-human CD11b Ab (eBioscience, San Diego, CA; catalog number 11-0118) for $30 \mathrm{~min}$ at $4^{\circ} \mathrm{C}$. At least 50,000 MNCs were gated based on forwardand side-scatter profiles, and further subdivided into

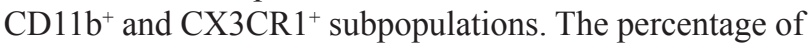
$\mathrm{CD}_{11} \mathrm{~b}^{+} \mathrm{CX} 3 \mathrm{CR}^{+}$cells was analyzed using a Navios flow cytometer (Beckman Coulter, Brea, CA; Figure 5).

\section{Statistical analysis}

Pearson's $\chi^{2}$ test or Fisher's exact test was used to analyze the association between the baseline $\mathrm{CD}_{11 \mathrm{~b}}{ }^{+} \mathrm{CX} 3 \mathrm{CR} 1^{+}$monocyte percentages and clinical variables, such as age, sex, Ann Arbor stage, serum LDH level, the ECOG performance status, presence of B symptoms, bulky disease, extranodal involvement, cell of origin by Hans classification [19], and the NCCNIPI [25]. To determine the optimal cutoffs for PB- and $\mathrm{BM}-\mathrm{CD} 11 \mathrm{~b}{ }^{+} \mathrm{CX} 3 \mathrm{CR} 1^{+}$cells and the ratio of $\mathrm{PB} / \mathrm{BM}-$ $\mathrm{CD} 11 \mathrm{~b}^{+} \mathrm{CX} 3 \mathrm{CR} 1^{+}$cells for predicting disease progression or death, receiver-operating-characteristics (ROC) curve analysis was used. ROC curve analysis demonstrated that the cutoffs of $3.68 \%(\mathrm{~PB}), 3.45 \%$ (BM), and 1.77 (PB/BM) had the best sensitivity and specificity to predict disease progression or death. Then, patients were categorized into high or low PB- or BM-CD $11 \mathrm{~b}^{+} \mathrm{CX} 3 \mathrm{CR} 1^{+}$monocyte groups according to the cutoffs. Spearman's correlation coefficient was used to assess the association between PB-CD $11 b^{+} \mathrm{CX} 3 \mathrm{CR} 1^{+}$and $\mathrm{BM}-\mathrm{CD} 11 \mathrm{~b}^{+} \mathrm{CX} 3 \mathrm{CR} 1^{+}$cells. PFS and OS were defined as the time from the date of diagnosis to the date of disease progression, death, or last follow-up, as appropriate, and were estimated using the Kaplan-Meier method. Differences in survival outcomes between two groups were tested using the log-rank test. A Cox proportional hazards regression model was used for univariate and multivariate analysis of PFS and OS. Variables with $P<0.05$ in the univariate analysis were included in the multivariate analysis; a forward conditional method was used, and the results were reported as a hazard ratio and $95 \% \mathrm{CI}$. A two-sided $P<0.05$ was considered statistically significant. All data analyses were carried out using SPSS software, version 19.0 (SPSS Inc., Chicago, IL).

\section{Abbreviations}

Bone marrow, BM; complete response, CR; computed tomography, CT; diffuse large B-cell lymphoma, DLBCL; lactate dehydrogenase, LDH; mononuclear cell, MNC; myeloid-derived suppressor cells, MDSCs; National Comprehensive Cancer Network-International Prognostic Index, NCCN-IPI; overall survival, OS; peripheral blood, PB; progression-free survival, PFS; 
receiver-operating-characteristics, ROC; Rituximab with cyclophosphamide, doxorubicin, vincristine, and prednisone, R-CHOP.

\section{Author contributions}

JYK designed the study, recruited patients, analyzed and interpreted data, and critically revised the manuscript; HYY and JAK designed the study, recruited patients, analyzed and interpreted data, and wrote the manuscript; HSK performed the statistical analysis and analyzed data; SHK and EY recruited patients and interpreted data; YP analyzed data and critically revised the manuscript.

\section{ACKNOWLEDGMENTS}

We thank all the patients who participated in and provided biological samples for this study.

\section{CONFLICTS OF INTEREST}

The authors have declared no conflicts of interest

\section{REFERENCES}

1. Kim JM, Ko YH, Lee SS, Huh J, Kang CS, Kim CW, Kang YK, Go JH, Kim MK, Kim WS, Kim YJ, Kim HJ, Kim HK, et al. WHO Classification of Malignant Lymphomas in Korea: Report of the Third Nationwide Study. Korean J Pathol. 2011; 45: 254-60.

2. Kwak JY. Treatment of diffuse large B cell lymphoma. Korean J Intern Med. 2012; 27: 369-77.

3. Lyden D, Hattori K, Dias S, Costa C, Blaikie P, Butros L, Chadburn A, Heissig B, Marks W, Witte L, Wu Y, Hicklin $\mathrm{D}, \mathrm{Zhu} \mathrm{Z}$, et al. Impaired recruitment of bone-marrowderived endothelial and hematopoietic precursor cells blocks tumor angiogenesis and growth. Nat Med. 2001; 7: 1194-201.

4. Yang L, DeBusk LM, Fukuda K, Fingleton B, Green-Jarvis B, Shyr Y, Matrisian LM, Carbone DP, Lin PC. Expansion of myeloid immune suppressor $\mathrm{Gr}+\mathrm{CD} 11 \mathrm{~b}+$ cells in tumorbearing host directly promotes tumor angiogenesis. Cancer Cell. 2004; 6: 409-21.

5. Clements DR, Sterea AM, Kim Y, Helson E, Dean CA, Nunokawa A, Coyle KM, Sharif T, Marcato P, Gujar SA, Lee PW. Newly recruited CD11b+, GR-1+, Ly6C(high) myeloid cells augment tumor-associated immunosuppression immediately following the therapeutic administration of oncolytic reovirus. J Immunol. 2015; 194: 4397-412.

6. Gabrilovich DI, Nagaraj S. Myeloid-derived suppressor cells as regulators of the immune system. Nat Rev Immunol. 2009; 9: 162-74.
7. Azzaoui I, Uhel F, Rossille D, Pangault C, Dulong J, Le Priol J, Lamy T, Houot R, Le Gouill S, Cartron G, Godmer P, Bouabdallah K, Milpied N, et al. T-cell defect in diffuse large B-cell lymphomas involves expansion of myeloidderived suppressor cells. Blood. 2016; 128: 1081-92.

8. Mantovani A, Marchesi F, Malesci A, Laghi L, Allavena P. Tumour-associated macrophages as treatment targets in oncology. Nat Rev Clin Oncol. 2017; 14:399-416.

9. Marmey B, Boix C, Barbaroux JB, Dieu-Nosjean MC, Diebold J, Audouin J, Fridman WH, Mueller CG, Molina TJ. CD14 and CD169 expression in human lymph nodes and spleen: specific expansion of CD14+CD169- monocytederived cells in diffuse large B-cell lymphomas. Hum Pathol. 2006; 37: 68-77.

10. Tadmor T, Bari A, Sacchi S, Marcheselli L, Liardo EV, Avivi I, Benyamini N, Attias D, Pozzi S, Cox MC, Baldini L, Brugiatelli M, Federico M, et al. Monocyte count at diagnosis is a prognostic parameter in diffuse large B-cell lymphoma: results from a large multicenter study involving 1191 patients in the pre- and post-rituximab era. Haematologica. 2014; 99: 125-30.

11. Wilcox RA, Ristow K, Habermann TM, Inwards DJ, Micallef IN, Johnston PB, Colgan JP, Nowakowski GS, Ansell SM, Witzig TE, Markovic SN, Porrata L. The absolute monocyte and lymphocyte prognostic score predicts survival and identifies high-risk patients in diffuse large-B-cell lymphoma. Leukemia. 2011; 25: 1502-9.

12. Porrata LF, Ristow KM, Habermann TM, Witzig TE, Colgan JP, Inwards DJ, Ansell SM, Micallef IN, Johnston PB, Nowakowski G, Thompson CA, Markovic SN. Peripheral blood absolute lymphocyte/monocyte ratio during rituximab, cyclophosphamide, doxorubicin, vincristine and prednisone treatment cycles predicts clinical outcomes in diffuse large B-cell lymphoma. Leuk Lymphoma. 2014; 55: 2728-38.

13. Kim JA, Kwak JY, Eunjung Y, Lee J, Park Y, Broxmeyer HE. Fractalkine/CX3CR1 Signaling Promotes Angiogenic Potentials in CX3CR1 Expressing Monocytes. Blood. 2016; 128: 2507.

14. Shireman PK. The chemokine system in arteriogenesis and hind limb ischemia. J Vasc Surg. 2007; 45: A48-56.

15. Hart KM, Bak SP, Alonso A, Berwin B. Phenotypic and functional delineation of murine CX(3)CR1 monocytederived cells in ovarian cancer. Neoplasia. 2009; 11: 56473, $1 \mathrm{p}$ following 73.

16. Hart KM, Usherwood EJ, Berwin BL. CX3CR1 delineates temporally and functionally distinct subsets of myeloidderived suppressor cells in a mouse model of ovarian cancer. Immunol Cell Biol. 2014; 92: 499-508.

17. Schmall A, Al-Tamari HM, Herold S, Kampschulte M, Weigert A, Wietelmann A, Vipotnik N, Grimminger F, Seeger W, Pullamsetti SS, Savai R. Macrophage and cancer cell cross-talk via CCR2 and CX3CR1 is a fundamental 
mechanism driving lung cancer. Am J Respir Crit Care Med. 2015; 191: 437-47.

18. Andreasson U, Ek S, Merz H, Rosenquist R, Andersen N, Jerkeman M, Dictor M, Borrebaeck CA. B cell lymphomas express CX3CR1 a non-B cell lineage adhesion molecule. Cancer Lett. 2008; 259: 138-45.

19. Hans CP, Weisenburger DD, Greiner TC, Gascoyne RD, Delabie J, Ott G, Muller-Hermelink HK, Campo E, Braziel RM, Jaffe ES, Pan Z, Farinha P, Smith LM, et al. Confirmation of the molecular classification of diffuse large B-cell lymphoma by immunohistochemistry using a tissue microarray. Blood. 2004; 103: 275-82.

20. Hart KM, Byrne KT, Molloy MJ, Usherwood EM, Berwin B. IL-10 immunomodulation of myeloid cells regulates a murine model of ovarian cancer. Front Immunol. 2011; 2: 29.

21. Sawanobori Y, Ueha S, Kurachi M, Shimaoka T, Talmadge JE, Abe J, Shono Y, Kitabatake M, Kakimi K, Mukaida $\mathrm{N}$, Matsushima K. Chemokine-mediated rapid turnover of myeloid-derived suppressor cells in tumor-bearing mice. Blood. 2008; 111: 5457-66.

22. Bazan JF, Bacon KB, Hardiman G, Wang W, Soo K, Rossi D, Greaves DR, Zlotnik A, Schall TJ. A new class of membrane-bound chemokine with a CX3C motif. Nature. 1997; 385: 640-4.

23. Imai $T$, Hieshima $K$, Haskell $C$, Baba M, Nagira M, Nishimura M, Kakizaki M, Takagi S, Nomiyama H, Schall TJ, Yoshie O. Identification and molecular characterization of fractalkine receptor CX3CR1, which mediates both leukocyte migration and adhesion. Cell. 1997; 91: 521-30.

24. Truman LA, Ford CA, Pasikowska M, Pound JD, Wilkinson SJ, Dumitriu IE, Melville L, Melrose LA, Ogden CA, Nibbs R, Graham G, Combadiere C, Gregory CD. CX3CL1/ fractalkine is released from apoptotic lymphocytes to stimulate macrophage chemotaxis. Blood. 2008; 112: 5026-36.

25. Zhou Z, Sehn LH, Rademaker AW, Gordon LI, Lacasce AS, Crosby-Thompson A, Vanderplas A, Zelenetz AD, Abel GA, Rodriguez MA, Nademanee A, Kaminski MS, Czuczman MS, et al. An enhanced International Prognostic Index (NCCN-IPI) for patients with diffuse large B-cell lymphoma treated in the rituximab era. Blood. 2014; 123: $837-42$.

26. Shen F, Zhang Y, Jernigan DL, Feng X, Yan J, Garcia FU, Meucci O, Salvino JM, Fatatis A. Novel Small-Molecule CX3CR1 Antagonist Impairs Metastatic Seeding and Colonization of Breast Cancer Cells. Mol Cancer Res. 2016; 14: 518-27.

27. Cheson BD, Pfistner B, Juweid ME, Gascoyne RD, Specht L, Horning SJ, Coiffier B, Fisher RI, Hagenbeek A, Zucca E, Rosen ST, Stroobants S, Lister TA, et al. Revised response criteria for malignant lymphoma. J Clin Oncol. 2007; 25: 579-86. 